\title{
The effects of acute stress on Pavlovian-instrumental transfer in rats
}

\author{
Steffi M. Pielock • Stephanie Braun • Wolfgang Hauber
}

Published online: 13 October 2012

(C) Psychonomic Society, Inc. 2012

\begin{abstract}
Pavlovian stimuli invigorate ongoing instrumental action, a phenomenon termed the Pavlovian-instrumental transfer (PIT) effect. Acute stressors can markedly enhance the release of corticotropin-releasing factor (CRF), and CRF injection into the nucleus accumbens increases the PIT effect. However, it is unknown whether acute stressors by themselves would amplify the PIT effect. Here, we examined the effects of acute stressors on PIT. Rats first received Pavlovian and instrumental training, and then the impact of the Pavlovian stimuli on instrumental responding was analyzed in the subsequent PIT test. Acute stressors were applied prior to the PIT test. Because the effects of acute stressors critically depend on stressor type and time of day, we used two acute stressors that involved one or several distinct stressors (denoted here as "single" vs. "multiple" stressors) applied either in the light or the dark period of the light:dark cycle. The results revealed that single and multiple stressors applied in the light period did not alter the PIT effect - that is, the ability of an appetitive Pavlovian stimulus to enhance leverpressing - or the basal leverpress rate. When applied in the dark period, single and multiple stressors also did not alter the PIT effect, but they did markedly reduce the basal leverpress rate. Diazepam pretreatment did not counteract the declines in basal instrumental responding in the PIT test that were induced by either a single or multiple stressors. Our findings suggest that acute stressors were unable to amplify the incentive salience of rewardpredictive Pavlovian stimuli to activate instrumental responding, but, depending on the time of day of stressor exposure, they did reduce basal instrumental responding.
\end{abstract}

\footnotetext{
S. M. Pielock $\cdot$ S. Braun $\cdot$ W. Hauber $(\triangle)$

Biologisches Institut, Abteilung Tierphysiologie,

Universität Stuttgart,

Pfaffenwaldring 57,

70550 Stuttgart, Germany

e-mail: hauber@bio.uni-stuttgart.de
}

Keywords Acute stress - Incentive motivation · Anxiety · Pavlovian-instrumental transfer · Rat

Pavlovian stimuli predictive of natural rewards such as food can increase leverpressing for food, a phenomenon termed the Pavlovian-instrumental transfer effect (PIT effect) (Estes, 1948; Lovibond, 1983). One important mechanism by which Pavlovian stimuli influence the vigor of an action is to create a general appetitive arousal that elevates instrumental responding (Rescorla \& Solomon, 1967). The central nucleus of the amygdala (Corbit \& Balleine, 2005; Holland \& Gallagher, 2003), the nucleus accumbens core (Corbit \& Balleine, 2011; Hall, Parkinson, Connor, Dickinson, \& Everitt, 2001), the ventral tegmental area (Corbit, Janak, \& Balleine, 2007; Murschall \& Hauber, 2006), and the mesoaccumbens dopamine system (Hall, et al., 2001; Lex \& Hauber, 2008; Wyvell \& Berridge, 2000) are major components of a neural circuitry mediating the PIT effect. Notably, Pecina, Schulkin, and Berridge (2006) demonstrated that the PIT effect was markedly enhanced not only by intraaccumbens amphetamine infusion but also by intraaccumbens infusion of corticotropin-releasing factor (CRF), and they concluded that activation of CRF subsystems in the nucleus accumbens - and, thus, acute stresscould amplify positive motivation for signaled rewards. Importantly, such paradoxical positive incentive effects could explain why acute stress can produce stimulustriggered bursts of binge eating, drug relapse, or other excessive pursuits of reward (Pecina et al., 2006).

It is well known that acute stress is associated with increased brain CRF and dopamine release (Bale \& Vale, 2004; Imperato, Puglisiallegra, Casolini, Zocchi, \& Angelucci, 1989). However, although CRF plays a crucial role (Bale \& Vale, 2004), it is important to note that acute stress responses involve the release of multiple stress mediators, including a number of other neuropeptides, such as 
vasopressin or dynorphin, as well as monoamine neurotransmitters and steroids (Joels \& Baram, 2009) and entail complex interacting circuits, in particular in the limbic forebrain, the hypothalamus, and the brainstem (Ulrich-Lai \& Herman, 2009). Because intra-accumbens CRF infusion may mimic the multifaceted neurochemical responses induced by acute stressors only to a minor extent, it is questionable whether acute stress would be able to amplify the PIT effect in a manner similar to intra-accumbens infusions of CRF. Therefore, in the present study we sought to explore the as-yet-unknown effects of acute stressors on PIT. Rats first received Pavlovian and instrumental training, and thereafter, the impact of the Pavlovian stimuli on instrumental responding was analyzed in a subsequent PIT test. Acute stressors were applied immediately prior to the PIT test. As the impact of acute stress critically depends on the stressor type and the time of day (Koolhaas et al., 2011), we included two distinct stressors that involved either one or several stressors (denoted here as "single" vs. "multiple" stressors) that were applied during the light or the dark period of the light: dark cycle. To assess hypothalamus-pituitary-adrenocortical (HPA) axis activity in response to these stressors, we also measured plasma corticosterone levels. Furthermore, we examined whether the standard anxiolytic drug diazepam would be able to reverse the impact of acute stressors on PIT.

\section{Materials and method}

All animal experiments were in compliance with the German Law of Animal Protection and were approved by the proper authorities.

\section{Subjects and apparatus}

Experimentally naive male Lister hooded rats (Harlan Winkelmann, Borchen, Germany) served as the subjects. The rats were housed in groups of four in transparent plastic cages $(55 \times 39 \times 27 \mathrm{~cm}$; Ferplast, Nürnberg, Germany) in a temperature- and humidity-controlled room $\left(20 \pm 2{ }^{\circ} \mathrm{C}\right.$, $50 \%-60 \%$ ) on a 12:12-h light:dark cycle (lights on at 7.00 a.m.; Exp. 1) or on an inverted 12:12-h light:dark cycle (lights off at 8.00 a.m.; Exps. 2 and 3). The rats had ad libitum access to water. Standard laboratory maintenance chow (Altromin, Lage, Germany) was given ad libitum for 5 days after arrival. Thereafter, food was restricted to $15 \mathrm{~g}$ per animal per day to maintain them at $\sim 85 \%$ of their freefeeding weight.

Training and testing took place in identical operant chambers $(24 \times 21 \times 30 \mathrm{~cm}$; Med Associates, St. Albans, VT $)$ housed within sound-attenuating cubicles. Each operant chamber was equipped with a pellet dispenser that delivered a 45-mg pellet into a receptacle that was positioned in the center of one wall. In addition, each chamber contained a retractable lever located on the left side of the receptacle. A 24-V/3-W houselight mounted at the top center of the opposite wall illuminated the chambers. The speaker that delivered the auditory conditioned stimuli was mounted on the wall opposite to the levers and the receptacle. Two auditory stimuli (white noise and a tone) served as conditioned stimuli. A computer system (MedPC Software; Med Associates) controlled the equipment and recorded the data.

Pavlovian-instrumental transfer

The testing for PIT consisted of three separate parts: instrumental training and Pavlovian training, followed by a transfer test in which the invigorating influences of the Pavlovian stimuli on instrumental behavior were determined in extinction. Each session started with the illumination of the houselight and the insertion of the appropriate lever, and each ended with the retraction of the lever and the turning off of the houselight.

Magazine training First, all subjects received one session of magazine training in order to habituate the animals to the operant chamber. During the magazine training, food pellets (45-mg pellets; Bio-Serv, Frenchtown, NJ) were delivered on a random-time (RT) 30-s schedule with no lever available.

Instrumental and Pavlovian training Thereafter, eight training days were given with two sessions per day, an instrumental training session in the morning and a Pavlovian training session in the afternoon. In the instrumental training sessions, the lever was inserted, and responding on the lever was reinforced on a random-interval (RI) schedule, starting with RI $2 \mathrm{~s}$ during the first session. For the next two sessions, the schedule was increased to RI $15 \mathrm{~s}$ and RI $30 \mathrm{~s}$, and for the remaining five sessions, to RI $60 \mathrm{~s}$; a session ended after $30 \mathrm{~min}$. The rats received Pavlovian training sessions with the lever retracted. An $80-\mathrm{dB}$ sound pressure level (SPL) auditory stimulus $(3-\mathrm{kHz}$ tone or white noise) served as the CS+ (conditioned stimulus, rewarded) in a counterbalanced fashion. Each session contained six 2min presentations of the $\mathrm{CS}+$, followed by an interstimulus interval (ISI) of 2-4 min. Food reward pellets were delivered only during the presentation of the CS+, on an RT-30-s schedule. Subsequent to this combined instrumentalPavlovian training, the animals received a single 30-min instrumental extinction session, with the lever available but not reinforced. In addition, one Pavlovian reminder was given. This session was similar to the Pavlovian training sessions, except that two additional, nonrewarded 2-min presentations of an $80-\mathrm{dB}$ SPL auditory $(3-\mathrm{kHz}$ tone or white noise) CSo stimulus (neutral stimulus, nonrewarded), 
as well as an additional ISI, were given preceding the fifth and following the sixth presentation of the $\mathrm{CS}+$. If the tone was the $\mathrm{CS}+$, the white noise was the CSo, and vice versa.

Transfer test The PIT effect was tested in extinction, while the lever was inserted into the operant chamber. Each stimulus (CS+ and CSo) was 2 min in duration and was preceded by a 2-min ISI period. The session always started with the presentation of the white noise, thus counterbalancing the order of the presentations of CS+ and CSo within each group, and the session ended after four presentations of each stimulus type (CS+ and CSo).

\section{Experimental design}

Three experiments were performed.

Experiments 1 and 2: We examined whether two distinct types of stressors, involving one or several prototypical types of acute stressors ("single" vs. "multiple" stressors; see below) and applied in either the light (Exp. 1) or the dark (Exp. 2) period of the light:dark cycle could alter the PIT effect measured in the transfer test. Instrumental and Pavlovian training and the transfer test were performed during either the light or the dark period; single or multiple stressors were applied to separate groups of rats immediately prior to the transfer test. Nonstressed rats served as the controls.

Experiment 3: Experiments 1 and 2 had revealed stressor effects on PIT when the stressors were applied during the dark, but not during the light, period. Therefore, in Experiment 3 we examined whether the observed darkperiod-related single- or multiple-stressor effects on the PIT test could be reduced by pretreatment with the standard anxiolytic drug diazepam. Nonstressed rats subjected to vehicle injections served as the controls.

\section{Types of acute stressors}

Single stressor This stressor consisted of acute restraint for $60 \mathrm{~min}$ in a brightly lit novel room (see, e.g., Reis et al., 2011; Wong et al., 2007): Rats were placed in air-accessible Plexiglas cylinders of 7-cm diameter and variable length, in order to accommodate different-sized animals.

Multiple stressor This stressor consisted of a combination of acute stressors, applied for $60 \mathrm{~min}$ in total in a brightly lit, novel room with music from a local radio station $(\sim 70 \mathrm{~dB}$ SPL), similar to the conditions used in previous studies (Chen et al., 2010). First, the rats were exposed to restraint for $20 \mathrm{~min}$ by placing them in Plexiglas cylinders. Thereafter, the rats were placed on an elevated platform
$(12 \times 12 \mathrm{~cm}, 1 \mathrm{~m}$ tall) for $20 \mathrm{~min}$ (Wong et al., 2007). Eventually, the rats were reexposed to restraint for $20 \mathrm{~min}$ by placing them back in the Plexiglas cylinders. During this restraint, five tail shocks $(1 \mathrm{~mA}, 1 \mathrm{~s})$ were applied (e.g., Waddell, Mallimo, \& Shors, 2010).

Rats were exposed to single or multiple stressors during the light or the dark phase of the light:dark cycle. Note that in those rats examined during the dark phase, stressor exposure in the bright room represented a light pulse that might act, per se, as an additional stressor (Song \& Rusak, 2000; Zubidat, Ben-Shlomo, \& Haim, 2007).

\section{Drug treatment}

Diazepam (1 mg/kg, i.p.; Sigma Aldrich, Steinheim, Germany) or vehicle ( $1 \mathrm{ml} / \mathrm{kg}$, i.p.) was given $90 \mathrm{~min}$ prior to the onset of PIT testing (i.e., $30 \mathrm{~min}$ before stressor exposure). Previous studies and our own pilot experiments had revealed that diazepam at $1 \mathrm{mg} / \mathrm{kg}$ i.p. produced anxiolytic-like effects devoid of marked locomotor inhibition (Boissier, Simon, \& Aron, 1968; Treit \& Fundytus, 1988) or suppression of instrumental responding (George, Hutson, \& Stephens, 2009). Due to sedative effects of this drug, higher doses reduced basal rates of leverpressing on their own (see also George et al., 2009). Diazepam was dissolved in a vehicle of $50 \%$ saline $(0.9 \% \mathrm{NaCl}), 10 \%$ pure ethanol, and $40 \%$ polyethylene glycol.

\section{Blood sampling and corticosterone analysis}

To assess the stressor effects in Experiments 1 and 2 on glucocorticoid secretion, we measured plasma corticosterone levels in separate groups of experimentally naive rats exposed to no, single, or multiple stressors in the light $(N=$ 3 , respectively) or in the dark ( $N=3$, respectively) period. In addition, to assess the stressor and drug effects in Experiment 3 on glucocorticoid secretion, plasma corticosterone levels were measured in experimentally naive rats subjected to vehicle administration and no stress or to diazepam administration and single or multiple stressors $(N=3$, respectively). Upon completion of the stressor exposure, the rats were anesthetized with an overdose of isoflurane (cppharma, Burgdorf, Germany) and decapitated. Short-term treatment with isoflurane per se, as used here, does not alter corticosterone levels (Zardooz, Rostamkhani, Zaringhalam, \& Faraji Shahrivar, 2010). Trunk blood was collected in a falcon tube $(50 \mathrm{ml})$ for $15 \mathrm{~min}$ and centrifuged $(4,000 \mathrm{rpm}$ for $10 \mathrm{~min}$; $\left.8{ }^{\circ} \mathrm{C}\right)$. Subsequently, the blood plasma was centrifuged $(4,000 \mathrm{rpm}$ for $4 \mathrm{~min}, \mathrm{RT})$ and stored at $-20^{\circ} \mathrm{C}$. Plasma corticosterone levels $(\mathrm{ng} / \mathrm{ml})$ were measured using the corticosterone ELISA kit RE52211 (IBL International GmbH, Hamburg, Germany). 
Data analysis

The data from the instrumental and Pavlovian training were subjected to an analysis of variance (ANOVA) for repeated measures, with Groups Exposed to Stressors as a betweensubjects factor and Training Day as a within-subjects factor. The PIT data were also subjected to an ANOVA for repeated measures, with Stressor Type as a between-subjects factor and Interval (ISI, CSo, or CS+) as a within-subjects factor. Post hoc analyses were performed using the least significant difference test. Plasma corticosterone values were also subjected to an ANOVA, with Stressor Type and Time of Day as between-subjects factors. The level of statistical significance was $p \leq .05$. All statistical computations were carried out with STATISTICA (Version 7.1; StatSoft, Inc., Tulsa, OK).

\section{Results}

Instrumental training

Experiment 1 Separate groups of rats to be exposed to no stressor $(n=22)$, a single stressor $(n=23)$, or multiple stressors $(n=20)$ were used; the rats were randomly assigned to their respective groups. Leverpress rates during instrumental training were similar across groups (Fig. 1a). An ANOVA revealed a significant effect of day $[F(7,427)=$ $190.61, p<.01]$ but not of group $[F(2,61)=1.73, p=.19]$, and no Day $\times$ Group interaction $[F(14,427)=1.60, p=.08]$.

Experiment 2 Separate groups of rats to be exposed to no stressor $(n=15)$, a single stressor $(n=12)$, or multiple stressors $(n=11)$ were used; the rats were randomly assigned to the respective groups. We found a significant effect of day $[F(7,245)=110.85, p<.01]$, a significant effect of group $[F$ $(2,35)=10.60, p<.01]$, and a trend toward a significant Day $\times$ Group interaction $[F(14,245)=1.62, p=.07]$ (Fig. 1b).

Experiment 3 Separate groups to be exposed to no stressor $(n=14)$, a single stressor $(n=12)$, or multiple stressors $(n=$ 12) were used; the rats were randomly assigned to the respective groups. An ANOVA revealed a significant effect of day $[F(7,245)=97.042, p<.01]$ and a significant Day $\times$ Group interaction $[F(14,245)=2.13, p=.01]$, but no effect of group $[F(2,35)=0.37, p=.69]$ (Fig. 1c).

The results from Experiments 2 and 3 indicated that the leverpress performance over days differed somewhat between groups during instrumental training. The reasons for the differences between groups are unknown, but they may not influence the PIT effect measured in the subsequent PIT test, for several reasons: (1) the approach ratios during Pavlovian training were similar across groups (see below); (2) the instrumental response rates during training did not predict the instrumental response rates in the PIT test performed in extinction; and (3) the PIT effect size does not critically depend on the instrumental response rate in the PIT test (Lex \& Hauber, 2008).

\section{Pavlovian training}

Stimulus-directed behavior during Pavlovian training was measured by calculating the approach ratio-that is, the percentages of time spent in the food receptacle during the $\mathrm{CS}+$ versus during the ISI-as follows: approach ratio = $(\% \mathrm{CS}+) /(\% \mathrm{CS}++\%$ ISI) (Hall et al., 2001).

Experiment 1 The approach ratios were similar in all groups (Fig. 2a). An ANOVA demonstrated a significant effects of day $[F(7,392)=6.48, p<.01]$, but no effect of group $[F(2$, $56)=0.79, p=.46]$ and no Day $\times$ Group interaction $[F(14$, $392)=0.54, p=.91]$.

Experiment 2 Likewise, in Experiment 2, the approach ratios of all groups were similar (Fig. 2b). An ANOVA showed a significant effect of day $[F(7,245)=3.65, p<$ $.01]$, but no effect of group $[F(2,35)=1.14, p=.33]$ and no Day $\times$ Group interaction $[F(14,245)=1.19, p=.28]$.

Experiment 3 Also, in Experiment 3 (Fig. 2c) the approach ratios increased to a similar extent in all groups, and an ANOVA revealed a significant effect of day $[F(7,238)=2.81, p<.01]$, but no significant group effect $[F(2,34)=0.20, p=.82]$ and no Day $\times$ Group interaction $[F(14,238)=1.24, p=.25]$.

\section{Transfer test}

Experiment 1 Presentation of the CS+ enhanced leverpressing relative to the ISI in both nonstressed control rats and rats exposed to single and to multiple acute stressors immediately before the transfer test. Furthermore, a minor decrease in overall response rates across all intervals was observed in rats subjected to multiple stressors, relative to control rats (Fig. 3a). An ANOVA demonstrated no effect of stressor type (no, single, or multiple stressors) $[F(2,61)=1.85, p=.17]$, a significant effect of interval $(\mathrm{CS}+, \mathrm{CSo}$, or ISI) $[F(2,122)=$ $55.99, p<.01]$, and no Interval $\times$ Stressor Type interaction $[F$ $(4,122)=1.94, p=.11]$. Post hoc comparisons further indicated higher responding in control rats during the CS+ relative to the ISI $(p<.05)$, but not during the CSo relative to the ISI $(p=.53)$. Likewise, in rats exposed to single and multiple stressors, leverpressing during the CS+ was higher relative to the ISI (single stressor, $p<.01$; multiple stressors, $p<.01$ ), but not during the CSo relative to the ISI (single stressor, $p<.23$; multiple stressors, $p=.69$ ). 
Fig. 1 Instrumental training: Mean leverpresses per minute ( \pm SEMs) during instrumental training sessions in

Experiments 1-3 (a-c) in control rats and in rats to be exposed to single or multiple stressors. Training was given during the light (Exp. 1) or during the dark (Exps. 2 and 3) period
A 20

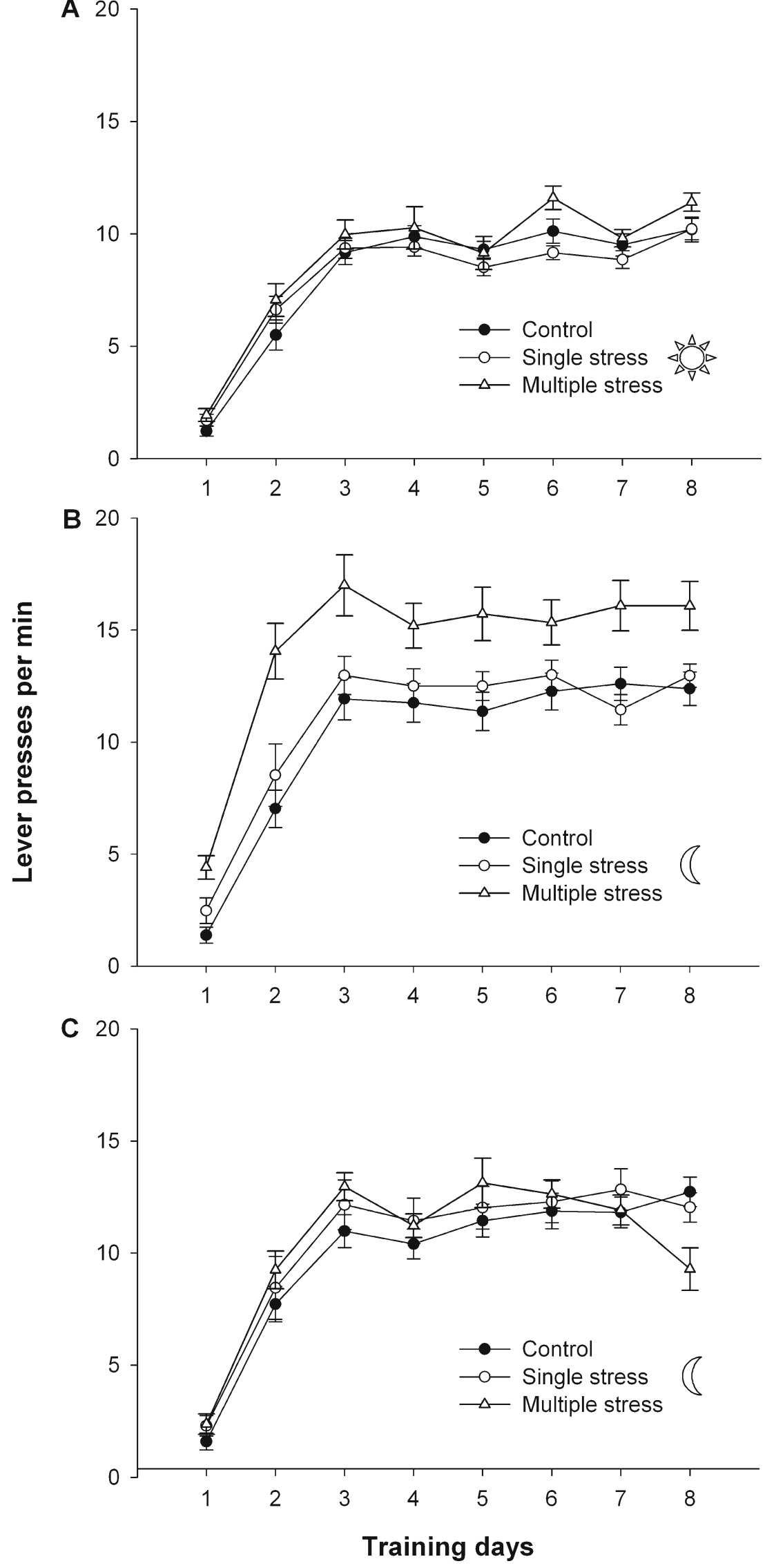


Fig. 2 Pavlovian training:

Mean approach ratios ( $\pm S E M \mathrm{~s})$

during Pavlovian training in

Experiments 1-3 (a-c) in control rats and in rats to be exposed to single or multiple stressors. Training was given during the light (Exp. 1) or during the dark (Exps. 2 and 3) period
A

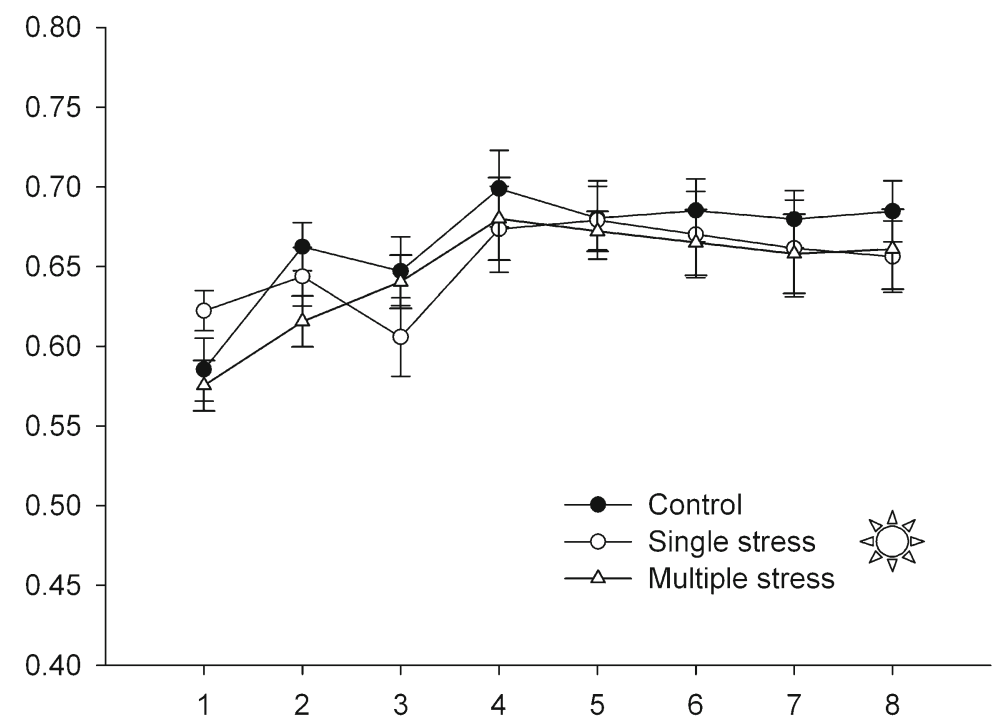

일

B $\quad 0.75$

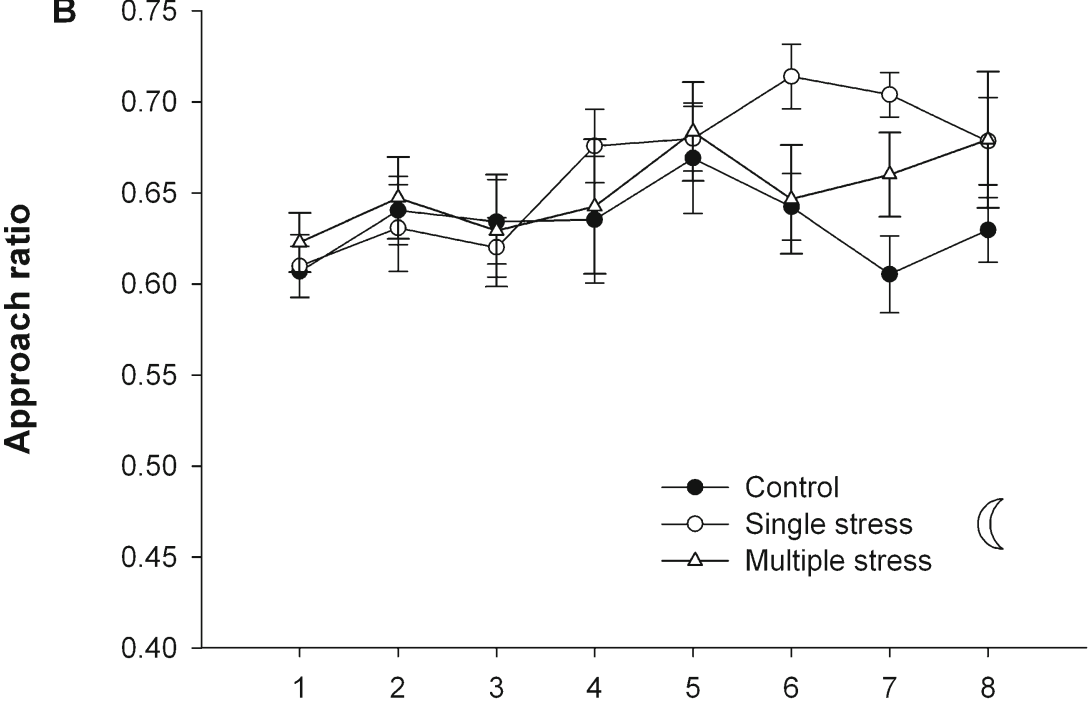

C

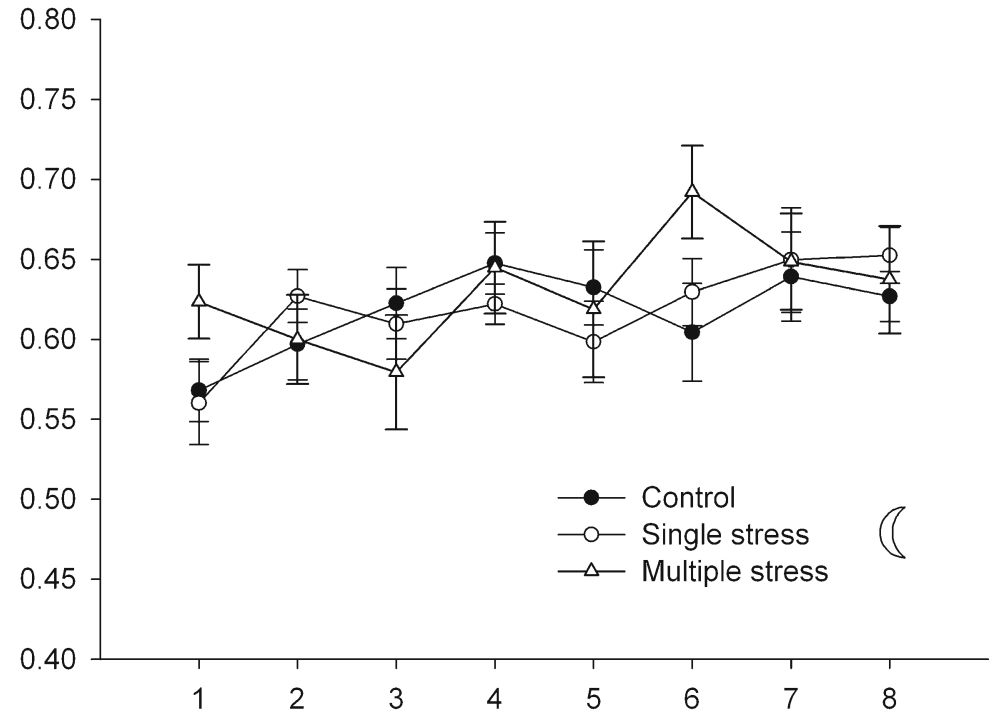

Training days 
Fig. 3 Pavlovian-instrumental transfer (PIT): Mean leverpresses per minute $( \pm S E M \mathrm{~s})$ in control rats and in rats exposed to single and multiple stressors immediately prior to the PIT test. In Experiment 1 (a), transfer testing was performed in the light period, and in Experiments 2 and 3 (b and $\mathbf{c}$ ), in the dark period. In Experiment 3, controls rats were pretreated with vehicle, and rats exposed to single or multiple stressors were pretreated with diazepam (DZ; $1 \mathrm{mg} / \mathrm{kg}$, i.p.). ${ }^{*} p<.05$ for comparisons of performance during the interstimulus interval (ISI), during the unconditioned, neutral stimulus (CSo), or during the conditioned stimulus $(\mathrm{CS}+)$

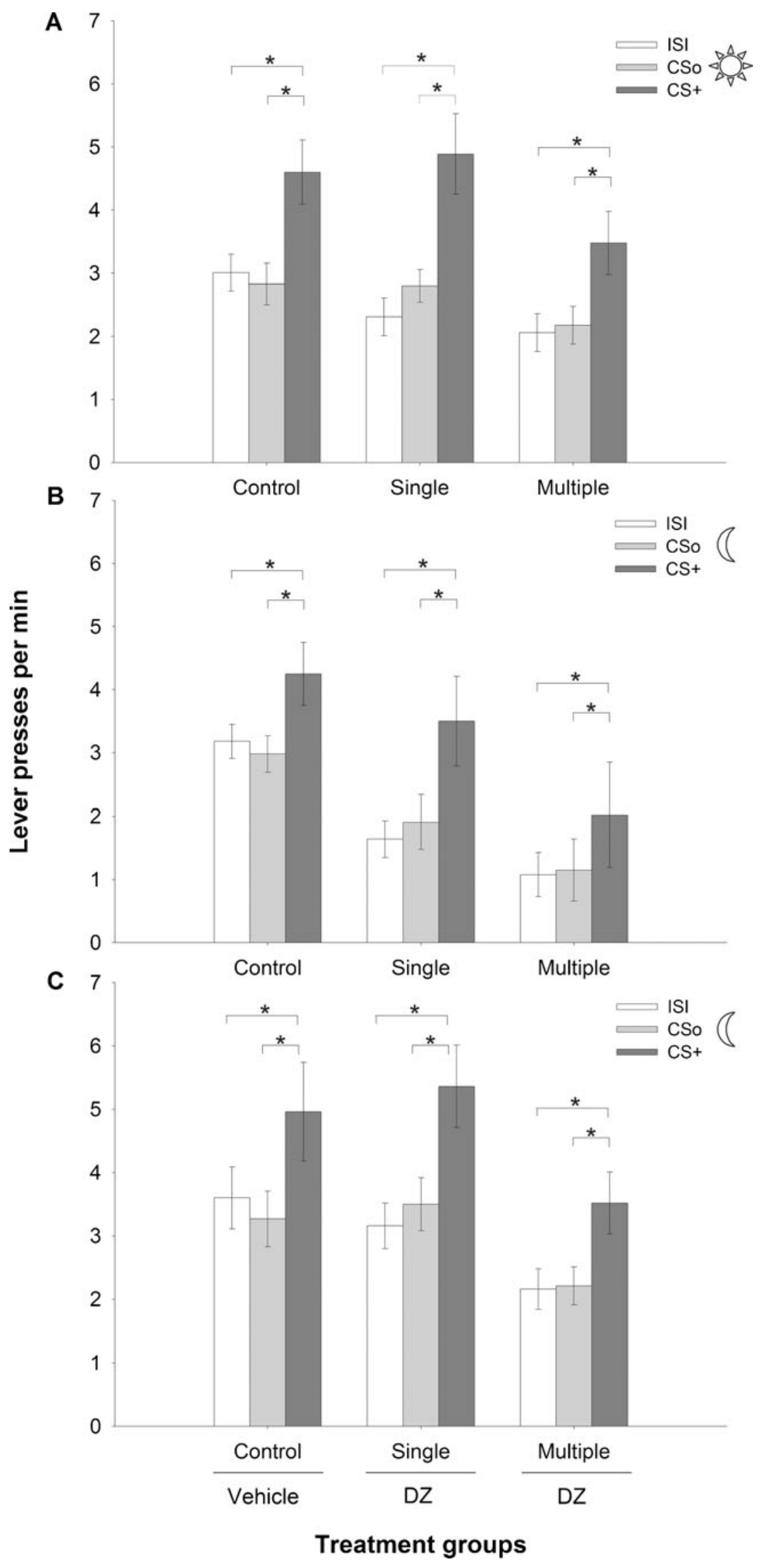


Experiment 2 This experiment demonstrated a PIT effect in both control rats and rats exposed to single and multiple stressors. However, baseline responding was lower in the rats exposed to single and multiple stressors, relative to control rats (Fig. 3b). Consistent with this notion, an ANOVA demonstrated significant effects of stressor type $[F(2,35)=7.05, p<.01]$ and interval $[F(2,70)=14.09, p$ $<.01]$, but no Interval $\times$ Stressor Type interaction $[F(4,70)$ $=0.62, p=.65]$. Post hoc comparisons further indicated higher responding during the CS+ relative to the ISI in control rats and in rats exposed to single and multiple stressors (controls, $p=.02$; single stressor, $p<.01$; multiple stressors, $p=.03$ ), but not during the CSo relative to the ISI (controls, $p=.65$; single stressor, $p=.63$; multiple stressor, $p=.87$ ). For a more detailed analysis of the treatment effect on overall leverpress rates, we performed separate pair-wise ANOVAs. The results revealed that the overall leverpress rates across all intervals (ISI, CS+, and CSo) were lower in the single-stressor group than in the control group $[F(1,25)$ $=5.97, p=.02]$ and also lower in the multiple-stressor group than in the control group $[F(1,24)=13.47, p=.01]$, but not between the single-stressor and multiple-stressor groups $[F$ $(1,21)=1.98, p=.17]$.

Experiment 3 A PIT effect was observed in the vehicletreated control rats exposed to no stressor, as well as in the diazepam-pretreated rats exposed to a single stressor and to multiple stressors (Fig. 3c). An ANOVA revealed significant effects of stressor type $[F(2,35)=3.26, p=.05]$ and interval $[F(2,70)=22.68, p<.01]$, but no Interval $\times$ Stressor Type interaction $[F(4,70)=0.61, p=.66]$. Post hoc comparisons further indicated higher responding in all treatment groups during the CS+ relative to the ISI (control, $p=.01$; single stressor, $p<.01$; multiple stressor, $p<.01$ ), but not during the CSo relative to the ISI (control, $p=.52$; single stressor, $p$ $=.55$; multiple stressor, $p=.88$ ). For a more detailed analysis, we analyzed the treatment effect on overall leverpress rates using separate pair-wise ANOVAs. The results revealed that the overall leverpress rates across intervals (ISI, CS+, and CSo) did not differ between the singlestressor and control groups $[F(1,24)=0.01, p=.92]$, but that they were lower in the multiple-stressor group than in the control group $[F(1,24)=4.37, p<.05]$, as well as in the single-stressor group $[F(1,22)=7.70, p=.01]$.

Collectively, the data from Experiments 2 and 3 point to the possibility that diazepam moderately reduced the stressor-induced decline in overall leverpress rates, in particular in rats exposed to the single stressor. To characterize the diazepam effects in more detail, we performed an ANOVA on the PIT effects measured in Experiments 2 and 3, with Experiment and Stressor Type as betweensubjects factors and Interval as a within-subjects factor. The results revealed significant effects of experiment $[F(1$,
$70)=11.06, p<.01]$, stressor type $[F(2,70)=8.80, p<.01]$, and interval $[F(2,140)=36.31, p<.01]$, but, importantly, no Experiment $\times$ Stressor Type interaction $[F(2,70)=1.14$, $p=.32]$, suggesting that diazepam had only marginal effects that failed to reach significance.

\section{Plasma corticosterone levels}

Corticosterone levels were higher in the untreated rats exposed to single and multiple stressors than in controls, and the effects of time of day seemed to be moderate (Table 1). In line with this notion, an ANOVA with Time of Day (light or dark period) and Stressor Type (no, single, or multiple stressors) as factors showed significant effects of stressor type $[F(2,12)=35.6, p<.01]$ and time of day $[F(1,12)=$ $5.3, p<.05]$, but no interaction between these factors. Post hoc comparisons indicated that in both the light and dark periods, corticosterone levels were increased in stressorexposed rats relative to control rats (Table 1). By contrast, corticosterone levels in the rats treated with vehicle or diazepam did not differ. An ANOVA with Stressor Type (no, single, or multiple stressors) as a factor revealed no significant treatment effects $[F(2,5)=1.81, p>.05]$. The corticosterone value of one diazepam-treated animal exposed to the single stressor was excluded, as it deviated from the group mean by more than $2 S D$ s.

\section{Discussion}

Here, we examined the impact of two types of acute stressors when they were applied in the light or the dark period of the light:dark cycle immediately prior to the PIT test. The main finding of our study was that both types of stressors applied in the light did not alter the PIT effect- that is, the ability of an appetitive Pavlovian stimulus to enhance

Table 1 Time of day, stressor, and drug effects on mean plasma corticosterone levels

\begin{tabular}{lllll}
\hline Time of Day & Stressor & Drug & Corticosterone $(\mathrm{ng} / \mathrm{ml})$ & $n$ \\
\hline Light period & Control & - & $195.4 \pm 72$ & 3 \\
Light period & Single & - & $738.6 \pm 36^{*}$ & 3 \\
Light period & Multiple & - & $1,053.3 \pm 99^{* \#}$ & 3 \\
Dark period & Control & - & $503.7 \pm 103$ & 3 \\
Dark period & Single & - & $902.8 \pm 101^{*}$ & 3 \\
Dark period & Multiple & - & $1,060.2 \pm 81^{*}$ & 3 \\
Dark period & Control & Vehicle & $661.9 \pm 233$ & 3 \\
Dark period & Single & Diazepam & $1,259.8 \pm 358$ & 2 \\
Dark period & Multiple & Diazepam & $896.8 \pm 36$ & 3 \\
& & & &
\end{tabular}

${ }^{*} p<.01$ vs. the respective control; ${ }^{*} p<.05$ single vs. multiple stressors 
leverpressing - and also left basal leverpress rates unchanged. By contrast, when applied in the dark period, both types of stressors did not amplify the PIT effect but did markedly reduce basal leverpress rates.

Acute stressor effects: Influence of time of day

In the light period, the acute single stressor stimulated the HPA axis, resulting in increased plasma corticosterone, as was shown by earlier studies (e.g., Keim \& Sigg, 1976; Porterfield et al., 2011; Ulrich-Lai et al., 2010), but it did not alter the PIT effect or basal instrumental response rates. Our pilot studies using other prototypical acute stressors applied in the light period - for instance, elevated platform stress (Howland \& Cazakoff, 2010) or extended restraint stress (Yuen et al., 2009)—gave similar results. Therefore, we employed a novel multiple stressor involving a combination of typical acute stressors used in previous studies (e.g., Howland \& Cazakoff, 2010; Keim \& Sigg, 1976; Musazzi et al., 2010; Yuen et al., 2009). Notably, exposure to the multiple stressor during the light period increased plasma corticosterone to a larger extent than did the single stressor applied during the light period, but it did not alter the PIT effect and instrumental response rates.

By contrast, in the dark period, single and multiple stressors elevated plasma corticosterone to about the same extent and significantly reduced instrumental response rates, but they left the PIT effect per se unaffected. This finding suggests that our acute stressors, which involved solely or predominately restraint, were, in behavioral terms, most effective in the dark period. This view is consistent with an earlier notion that, for rats, stress procedures involving restraint elicit more pronounced physiological and behavioral effects during the dark (activity) period than during the light (inactivity) period (Koolhaas et al., 2011). As was pointed out by the authors of this previous study, from an ecological point of view this observation is not surprising, as, for instance, during the light (inactivity) period feral Norway rats hide in narrow burrow systems.

Consistent with earlier reports (e.g., Atkinson \& Waddell, 1997; Chacon et al., 2005; Gallo \& Weinberg, 1981), plasma corticosterone levels in our rats were generally higher during the dark period than during the light period. Moreover, plasma corticosterone elevations after single or multiple stressor correspond to those reported in previous studies using similar or other acute stressors (e.g., Kavushansky, Klein, \& Ben-Shachar, 2010; Kavushansky \& Richter-Levin, 2006; Ulrich-Lai \& Herman, 2009; Woodson, Macintosh, Fleshner, \& Diamond, 2003). The plasma corticosterone elevations reported in previous studies were not or were only partially related to stressor type or intensity, the timing of the stress exposure, or the magnitude of the stress-induced behavioral effects. For instance, male rats exposed to a predator such as a cat or to a female receptive rat showed equivalent increases in plasma corticosterone levels, but only cat-exposed rats exhibited working memory deficits (Woodson et al., 2003). Furthermore, the effects of acute restraint stress on cost/benefit decision making in rats were not mimicked by systemic administration of corticosterone, suggesting that increased corticosterone levels may be not sufficient to impair this form of behavioral choice (Shafiei, Gray, Viau, \& Floresco, 2012). Overall, these findings suggest that plasma corticosterone levels may not always be a sensitive index of stress intensity (e.g., Natelson et al., 1987). Correspondingly, our present findings provide strong support for the notion that, in rats, the behavioral effects of acute stressors involving restraint critically depend on the time of day, but depend less on stressor intensity.

\section{Acute stress effects on PIT}

A major mechanism by which Pavlovian stimuli might influence the vigor of an action is to create a general appetitive arousal that elevates instrumental responding (e.g., Rescorla \& Solomon, 1967). Notably, this PIT effect has been markedly enhanced not only by intra-accumbens amphetamine infusion (Wyvell \& Berridge, 2000) but also by intra-accumbens CRF infusion, suggesting that the activation of CRF subsystems in the nucleus accumbens and, by implication, acute stress could amplify positive motivation for signaled rewards (Pecina et al., 2006). Importantly, such paradoxical positive incentive effects could explain why acute stress sometimes produces stimulus-triggered bursts of binge eating, drug relapse, or other excessive pursuits of reward (Dallman, Pecoraro, Houshyar, \& Griffith, 2005; Marinelli \& Piazza, 2002). Contrasting with this view, our results indicate that, unlike intra-accumbens CRF, acute stressors applied prior the PIT test did not enhance the ability of an appetitive CS + to invigorate instrumental responding, but rather impaired basal instrumental responding for an expected reward. As only high but not low doses of intra-accumbens CRF have been effective (Pecina et al., 2006), the failure to observe an amplified PIT effect could be due simply to subthreshold accumbens CRF levels induced by the acute stressors used here. To our knowledge, the effects of the stressors used here on intra-accumbens CRF levels are unknown. Microdialysis studies have revealed that restraint stress for 20 min produced a threefold increase of CRF levels in limbic brain areas such as the amygdala (Merlo Pich et al., 1995), and possibly in the nucleus accumbens as well; however, direct evidence for the latter claim is missing. Furthermore, restraint stress for $90 \mathrm{~min}$ markedly increased intra-accumbens dopamine release (Imperato et al., 1989), indicating that the stressors used in the present study can produce marked neurochemical effects. 
Furthermore, our observation that both single and multiple stressors applied in the dark period reduced basal leverpressing suggests that the stressors were behaviorally effective.

Importantly, acute stress responses involve the release of multiple stress mediators, including a number of other neuropeptides, such as vasopressin or dynorphin, as well as monoamine neurotransmitters and steroids (Joels \& Baram, 2009). Furthermore, acute stress responses are mediated by complex neural circuits in the limbic forebrain, the hypothalamus, and the brainstem (Ulrich-Lai \& Herman, 2009). Thus, an intra-accumbens CRF infusion may mimic the multifaceted neurochemical responses in many brain areas induced by acute stressors only to a minor extent. For instance, it is conceivable that acute stressors activate the CRF subsystems that mediate aversive states (Schulkin, Morgan, \& Rosen, 2005), possibly counteracting the expression of an amplified PIT effect governed by accumbens CRF subsystems. Overall, our finding that acute stressors were unable to amplify the incentive salience of a rewardpredictive $\mathrm{CS}+$ in order to activate reward-directed responding puts in question a general role of acute stressors in precipitating paradoxical incentive effects-for instance, stimulus-triggered bursts of binge eating or drug relapse (Pecina et al., 2006). Yet, it is important to note that acute stressor effects depend on multiple variables; thus, other types of expected rewards, such as drugs, could enhance the incentive motivation triggered by an appetitive $\mathrm{CS}+$, in particular in rats chronically pretreated with addictive drugs. Furthermore, the fact that the PIT effect was insensitive to acute stressors implies that the retrieval of well-learned memories for CS-US associations, unlike other forms of memory (de Kloet, Oitzl, \& Joels, 1999), and the behavioral impact mediated by the appetitive CS + seem to be relatively resistant to acute stress. To some extent, the resistance of the PIT effect to acute stressors may be explained by Pavlovian training over not less than 8 days, as it has been implemented in most PIT protocols (Holmes, Marchand, \& Coutureau, 2010). In other words, it is possible that the PIT effect could become sensitive to acute stressors if a PIT protocol with more limited Pavlovian training would be used.

Importantly, our results further show that acute stressors significantly reduced basal instrumental responding for an expected reward during the PIT test. As acute stressors can compromise performance of skilled paw movements (Metz, Jadavji, \& Smith, 2005), the reduced leverpressing seen here could simply reflect impaired motor function per se. However, such a chronic, state-like motor impairment should have applied for the entire transfer test, regardless of whether or not the CS+ stimulus was present. By contrast, we observed that presentation of an appetitive CS+ was still able to invigorate leverpressing, an observation that seems to rule out basic motor impairments as an explanation for reduced instrumental responding. Considerable evidence suggests that, rather than reducing reward-seeking behavior, exposure to an acute stressor can have the opposite effect, stimulating palatable food intake or reinstating drug-taking behavior (Cador et al., 1992; Kalivas, Duffy, \& Latimer, 1987; Stewart, 2003). In turn, pleasurable behaviors such as palatable food intake were able to reduce neuroendocrine, cardiovascular, and behavioral responses to acute stress (Ulrich-Lai \& Herman, 2009). By contrast, a large number of studies have suggested that both acute stress and intracerebroventricular CRF inhibited food intake and behaviors associated with food intake. For instance, CRF decreased food-maintained instrumental responding under various reinforcement schedules, including differential reinforcements of low rate or combined fixed-interval and fixed-ratio schedules (see Glowa, Barrett, Russell, \& Gold, 1992, for a review). Remarkably, depending on a number of variables, such as stress intensity or context, acute stress can either reinstate or inhibit drug seeking (Lu, Shepard, Hall, \& Shaham, 2003; Shaham, Erb, \& Stewart, 2000). Furthermore, it is well documented that acute stress can produce fear and anxiety (Davis, 2006; Schulkin et al., 2005). For instance, by producing anxiety, a footshockpredictive stimulus can markedly suppress instrumental responding (Blanchard \& Blanchard, 1969; Hunt \& Brady, 1951). These findings point to the possibility that stressinduced anxiety could have contributed to the reduced instrumental responding observed here. However, our data do not provide support to this notion, as the standard anxiolytic drug diazepam at a dose of $1 \mathrm{mg} / \mathrm{kg}$ only marginally reduced leverpressing after stressor exposition. Notably, in acutestress paradigms, low doses of diazepam $(<3 \mathrm{mg} / \mathrm{kg})$ can produce anxiolytic effects on a variety of behavioral measures (Grahn, Kalman, Brennan, Watkins, \& Maier, 1995; Kalman, Kim, Cole, Chi, \& Spencer, 1997) without decreasing stress-induced rises in plasma corticosterone (Keim \& Sigg, 1976). Nevertheless, we cannot exclude the possibility that higher doses of diazepam could have antagonized stress-induced behavioral effects. However, our pilot studies indicated that higher doses reduced basal rates of leverpressing on their own. Likewise, a study by George et al. (2009) demonstrated that, due to sedative effects, diazepam at doses of 3 and $10 \mathrm{mg} / \mathrm{kg}$ reduced basal rates of leverpresses in the PIT test, an effect not seen with a dose of $1 \mathrm{mg} / \mathrm{kg}$, as used here. Thus, anxiolytic or antidepressant drugs with a lower incidence of sedative effects could be an option for future studies. Taken together, our findings clearly show that acute stressors impaired basal instrumental responding; however, the underlying behavioral mechanisms remain to be determined.

\section{Conclusions}

Our study demonstrated for the first time that acute stressors did not amplify the PIT effect, but rather reduced basal 
instrumental responding during the PIT test, an effect that critically depended on the time of day of stressor exposure. Importantly, these findings suggest that, unlike intraaccumbens CRF (Pecina et al., 2006), acute stress was unable to enhance the incentive salience of a CS+ that was predictive of natural reward so as to invigorate rewarddirected responding, but instead impaired basal instrumental responding.

Author note This research was supported by Deutsche Forschungsgemeinschaft Grant No. HA2340/6-3. We thank our colleagues F. Wollnik and E. Scheibler for technical advice and scientific discussions, as well as I. Nevar for assistance in animal husbandry. The authors declare no conflict of interest. The authors' contributions were as follows: S.M.P. participated in the research design, performed behavioral testing and data analysis, and drafted the manuscript; S.B. developed the stress protocols; and W.H. participated in the research design and data analysis and revised the manuscript.

\section{References}

Atkinson, H. C., \& Waddell, B. J. (1997). Circadian variation in basal plasma corticosterone and adrenocorticotropin in the rat: Sexual dimorphism and changes across the estrous cycle. Endocrinology, 138, 3842-3848.

Bale, T. L., \& Vale, W. W. (2004). CRF and CRF receptors: Role in stress responsivity and other behaviors. Annual Review of Pharmacology and Toxicology, 44, 525-557.

Blanchard, R. J., \& Blanchard, D. C. (1969). Passive and active reactions to fear-eliciting stimuli. Journal of Comparative Physiology and Psychology, 68, 129-135.

Boissier, J. R., Simon, P., \& Aron, C. (1968). A new method for rapid screening of minor tranquillizers in mice. European Journal of Pharmacology, 4, 145-151.

Cador, M., Dumas, S., Cole, B. J., Mallet, J., Koob, G. F., Lemoal, M., \& Stinus, L. (1992). Behavioral sensitization induced by psychostimulants or stress-Search for a molecular-basis and evidence for a crf-dependent phenomenon. Annals of the New York Academy of Sciences, 654, 416-420.

Chacon, F., Esquifino, A. I., Perello, M., Cardinali, D. P., Spinedi, E., \& Alvarez, M. P. (2005). 24-hour changes in ACTH, corticosterone, growth hormone, and leptin levels in young male rats subjected to calorie restriction. Chronobiology International, 22, 253-265. doi:10.1081/cbi-200053522

Chen, Y., Rex, C. S., Rice, C. J., Dube, C. M., Gall, C. M., Lynch, G., \& Baram, T. Z. (2010). Correlated memory defects and hippocampal dendritic spine loss after acute stress involve corticotropin-releasing hormone signaling. Proceedings of the National Academy of Sciences, 107, 13123-13128. doi:10.1073/pnas.1003825107

Corbit, L. H., \& Balleine, B. W. (2005). Double dissociation of basolateral and central amygdala lesions on the general and outcomespecific forms of Pavlovian-instrumental transfer. Journal of Neuroscience, 25, 962-970.

Corbit, L. H., \& Balleine, B. W. (2011). The general and outcomespecific forms of Pavlovian-instrumental transfer are differentially mediated by the nucleus accumbens core and shell. Journal of Neuroscience, 31, 11786-11794. doi:10.1523/JNEUROSCI.271111.2011
Corbit, L. H., Janak, P. H., \& Balleine, B. W. (2007). General and outcome-specific forms of Pavlovian-instrumental transfer: The effect of shifts in motivational state and inactivation of the ventral tegmental area. European Journal of Neuroscience, 26, 31413149 .

Dallman, F., Pecoraro, C., Houshyar, H., \& Griffith, K. (2005). Stress, glucocorticoids and obesity. Behavioural Pharmacology, 16, S13-S13.

Davis, M. (2006). Neural systems involved in fear and anxiety measured with fear-potentiated startle. American Psychologist, 61, 741-756. doi:10.1037/0003-066X.61.8.741

de Kloet, E. R., Oitzl, M. S., \& Joels, M. (1999). Stress and cognition: Are corticosteroids good or bad guys? Trends in Neurosciences, 22, 422-426.

Estes, W. K. (1948). Discriminative conditioning: II. Effects of a Pavlovian conditioned stimulus upon a subsequently established operant response. Journal of Experimental Psychology, 38, 173177. doi: $10.1037 / \mathrm{h} 0057525$

Gallo, P. V., \& Weinberg, J. (1981). Corticosterone rhythmicity in the rat-Interactive effects of dietary restriction and schedule of feeding. Journal of Nutrition, 111, 208-218.

George, S. A., Hutson, P. H., \& Stephens, D. N. (2009). Differential effects of MPEP and diazepam in tests of conditioned emotional response and Pavlovian-to-instrumental transfer suggests "anxiolytic" effects are mediated by different mechanisms. Psychopharmacology, 204, 499-509. doi:10.1007/s00213-0091479-6

Glowa, J. R., Barrett, J. E., Russell, J., \& Gold, P. W. (1992). Effects of corticotropin releasing hormone on appetitive behaviors. Peptides, 13, 609-621.

Grahn, R. E., Kalman, B. A., Brennan, F. X., Watkins, L. R., \& Maier, S. F. (1995). The elevated plus-maze is not sensitive to the effect of stressor controllability in rats. Pharmacology Biochemistry and Behavior, 52, 565-570.

Hall, J., Parkinson, J. A., Connor, T. M., Dickinson, A., \& Everitt, B. J. (2001). Involvement of the central nucleus of the amygdala and nucleus accumbens core in mediating Pavlovian influences on instrumental behaviour. European Journal of Neuroscience, 13, 1984-1992.

Holland, P. C., \& Gallagher, M. (2003). Double dissociation of the effects of lesions of basolateral and central amygdala on conditioned stimulus-potentiated feeding and Pavlovian-instrumental transfer. European Journal of Neuroscience, 17, 1680-1694.

Holmes, N. M., Marchand, A. R., \& Coutureau, E. (2010). Pavlovian to instrumental transfer: A neurobehavioural perspective. Neuroscience and Biobehavioral Reviews, 34, 1277-1295.

Howland, J. G., \& Cazakoff, B. N. (2010). Effects of acute stress and GluN2B-containing NMDA receptor antagonism on object and object-place recognition memory. Neurobiology of Learning and Memory, 93, 261-267. doi:10.1016/j.nlm.2009.10.006

Hunt, H. F., \& Brady, J. V. (1951). Some effects of electro-convulsive shock on a conditioned emotional response (anxiety). Journal of Comparative and Physiological Psychology, 44, 88-98.

Imperato, A., Puglisiallegra, S., Casolini, P., Zocchi, A., \& Angelucci, L. (1989). Stress-induced enhancement of dopamine and acetylcholine-release in limbic structures - Role of corticosterone. European Journal of Pharmacology, 165, 337-338.

Joels, M., \& Baram, T. Z. (2009). OPINION: The neuro-symphony of stress. Nature Reviews Neuroscience, 10, U459-U484. doi:10.1038/nrn2632

Kalivas, P. W., Duffy, P., \& Latimer, L. G. (1987). Neurochemical and behavioral effects of corticotropin-releasing factor in the ventral tegmental area of the rat. Journal of Pharmacology and Experimental Therapeutics, 242, 757-763.

Kalman, B. A., Kim, P. J., Cole, M. A., Chi, M. S., \& Spencer, R. L. (1997). Diazepam attenuation of restraint stress-induced 
corticosterone levels is enhanced by prior exposure to repeated restraint. Psychoneuroendocrinology, 22, 349-360.

Kavushansky, A., Klein, E., \& Ben-Shachar, D. (2010). A possible involvement of opioid-degrading enzymes in individual reactivity to acute traumatic stress. European Neuropsychopharmacology, 20, S41-S42.

Kavushansky, A., \& Richter-Levin, G. (2006). Effects of stress and corticosterone on activity and plasticity in the amygdala. Journal of Neuroscience Research, 84, 1580-1587. doi:10.1002/jnr.21058

Keim, K. L., \& Sigg, E. B. (1976). Physiological and biochemical concomitants of restraint stress in rats. Pharmacol Biochemistry and Behavior, 4, 289-297.

Koolhaas, J. M., Bartolomucci, A., Buwalda, B., de Boer, S. F., Flugge, G., Korte, S. M., . . . Fuchs, E. (2011). Stress revisited: A critical evaluation of the stress concept. Neuroscience and Biobehavioral Reviews, 35, 1291-1301. doi:10.1016/ j.neubiorev.2011.02.003

Lex, A., \& Hauber, W. (2008). Dopamine D1 and D2 receptors in the nucleus accumbens core and shell mediate Pavlovian-instrumental transfer. Learning \& Memory, 15, 483-491.

Lovibond, P. F. (1983). Facilitation of instrumental behavior by a Pavlovian appetitive conditioned stimulus. Journal of Experimental Psychology: Animal Behavior Processes, 9, 225-247. doi:10.1037/00977403.9.3.225

Lu, L., Shepard, J. D., Hall, F. S., \& Shaham, Y. (2003). Effect of environmental stressors on opiate and psychostimulant reinforcement, reinstatement and discrimination in rats: A review. Neuroscience and Biobehavioral Reviews, 27, 457-491. doi:10.1016/S0149-7634(03)00073-3

Marinelli, M., \& Piazza, P. V. (2002). Interaction between glucocorticoid hormones, stress and psychostimulant drugs. European Journal of Neuroscience, 16, 387-394

Merlo Pich, E., Lorang, M., Yeganeh, M., Rodriguez de Fonseca, F., Raber, J., Koob, G. F., \& Weiss, F. (1995). Increase of extracellular corticotropin-releasing factor-like immunoreactivity levels in the amygdala of awake rats during restraint stress and ethanol withdrawal as measured by microdialysis. Journal of Neuroscience, 15, 5439-5447.

Metz, G. A., Jadavji, N. M., \& Smith, L. K. (2005). Modulation of motor function by stress: A novel concept of the effects of stress and corticosterone on behavior. European Journal of Neuroscience, 22, 1190-1200. doi:10.1111/j.1460-9568.2005.04285.x

Murschall, A., \& Hauber, W. (2006). Inactivation of the ventral tegmental area abolished the general excitatory influence of Pavlovian cues on instrumental performance. Learning \& Memory, 13, 123-126.

Musazzi, L., Milanese, M., Farisello, P., Zappettini, S., Tardito, D., Barbiero, V. S., \& Popoli, M. (2010). Acute stress increases depolarization-evoked glutamate release in the rat prefrontal/ frontal cortex: The dampening action of antidepressants. Plos ONE, 5, 8566. doi:10.1371/journal.pone.0008566

Natelson, B. H., D. Creighton, et al. (1987). Adrenal hormonal indices of stress in laboratory rats. Physiology \& Behavior 39(1):117-125.

Pecina, S., Schulkin, J., \& Berridge, K. C. (2006). Nucleus accumbens corticotropin-releasing factor increases cue-triggered motivation for sucrose reward: Paradoxical positive incentive effects in stress? BMC Biology, 4, 8.

Porterfield, V. M., Zimomra, Z. R., Caldwell, E. A., Camp, R. M., Gabella, K. M., \& Johnson, J. D. (2011). Rat strain differences in restraint stress-induced brain cytokines. Neuroscience, 188, 4854. doi:10.1016/j.neuroscience.2011.05.023

Reis, D. G., A. A. Scopinho, et al. (2011). Behavioral and autonomic responses to acute restraint stress are segregated within the lateral septal area of rats. PLoS ONE 6(8):e23171.
Rescorla, R. A., \& Solomon, R. L. (1967). Two-process learning theory: Relationships between Pavlovian conditioning and instrumental learning. Psychological Review, 74, 151-182. doi:10.1037/h0024475

Schulkin, J., Morgan, M. A., \& Rosen, J. B. (2005). A neuroendocrine mechanism for sustaining fear. Trends in Neurosciences, 28, 629 635. doi:10.1016/j.tins.2005.09.009

Shafiei, N., Gray, M., Viau, V., \& Floresco, S. B. (2012). Acute stress induces selective alterations in cost/benefit decision-making. Neuropsychopharmacology, 37, 2194-2209. doi:10.1038/npp. 2012.69

Shaham, Y., Erb, S., \& Stewart, J. (2000). Stress-induced relapse to heroin and cocaine seeking in rats: A review. Brain Research Reviews, 33, 13-33.

Song, X., \& Rusak, B. (2000). Acute effects of light on body temperature and activity in Syrian hamsters: Influence of circadian phase. American Journal of Physiology: Regulatory, Integrative and Comparative Physiology, 278, R1369-R1380.

Stewart, J. (2003). State and trait changes in motivational systems control: The re-initiation of drug seeking after abstinence. Behavioural Pharmacology, 14, S23-S23.

Treit, D., \& Fundytus, M. (1988). Thigmotaxis as a test for anxiolytic activity in rats. Pharmacology Biochemistry and Behavior, 31, 959-962.

Ulrich-Lai, Y. M., Christiansen, A. M., Ostrander, M. M., Jones, A. A., Jones, K. R., Choi, D. C., \& Herman, J. P. (2010). Pleasurable behaviors reduce stress via brain reward pathways. Proceedings of the National Academy of Sciences, 107, 20529-20534. doi:10.1073/pnas.1007740107

Ulrich-Lai, Y. M., \& Herman, J. P. (2009). Neural regulation of endocrine and autonomic stress responses. Nature Reviews Neuroscience, 10, 397-409. doi:10.1038/nrn2647

Waddell, J., Mallimo, E., \& Shors, T. (2010). d-cycloserine reverses the detrimental effects of stress on learning in females and enhances retention in males. Neurobiology of Learning and Memory, 93, 31-36. doi:10.1016/j.nlm.2009.08.002

Wong, T. P., Howland, J. G., Robillard, J. M., Ge, Y., Yu, W., Titterness, A. K., . . . Wang, Y. T. (2007). Hippocampal longterm depression mediates acute stress-induced spatial memory retrieval impairment. Proceedings of the National Academy of Sciences, 104, 11471-11476. doi:10.1073/pnas.0702308104

Woodson, J. C., Macintosh, D., Fleshner, M., \& Diamond, D. M. (2003). Emotion-induced amnesia in rats: Working memory-specific impairment, corticosterone-memory correlation, and fear versus arousal effects on memory. Learning \& Memory, 10, 326-336. doi:10.1101/1m.62903

Wyvell, C. L., \& Berridge, K. C. (2000). Intra-accumbens amphetamine increases the conditioned incentive salience of sucrose reward: Enhancement of reward "wanting" without enhanced "liking" or response reinforcement. Journal of Neuroscience, 20, 8122-8130.

Yuen, E. Y., Liu, W. H., Karatsoreos, I. N., Feng, J., McEwen, B. S., \& Yan, Z. (2009). Acute stress enhances glutamatergic transmission in prefrontal cortex and facilitates working memory. Proceedings of the National Academy of Sciences, 106, 14075-14079. doi:10.1073/pnas.0906791106

Zardooz, H., Rostamkhani, F., Zaringhalam, J., \& Faraji Shahrivar, F. (2010). Plasma corticosterone, insulin and glucose changes induced by brief exposure to isoflurane, diethyl ether and $\mathrm{CO}_{2}$ in male rats. Physiological Research, 59, 973-978.

Zubidat, A. E., Ben-Shlomo, R., \& Haim, A. (2007). Thermoregulatory and endocrine responses to light pulses in short-day acclimated social voles (Microtus socialis). Chronobiology International, 24, 269-288. doi:10.1080/07420520701284675 